\title{
The evolvement of co-production of Biodegradable biological material PHB
}

\author{
Shuang Gao ${ }^{1 *}$, Jiao Feng ${ }^{1}$, Lihua Sun ${ }^{1}$, Hong $\operatorname{Tian}^{2,1}$ \\ ${ }^{1}$ Biological Engineering Department, Liaoning Economic Management Cadre Institute, Shenyang, \\ 110122, P. R. China \\ ${ }^{2}$ College of Chemistry and Life Sciences, Shenyang Normal University, Shenyang, 110034, P. R. \\ China \\ * Corresponding author: Biological Engineering Department, Liaoning Economic Management \\ Cadre Institute, Shenyang, 110122, P. R. China. Tel: 86-24-89874326.
}

E-mail address: Ingaos@126.com

\begin{abstract}
Keywords: Poly- $\beta$-hydroxybutyrate, Ectoine, Co-production, Production cost.
Abstract. Petrochemical industry plastics is widely used in the life. Because of its non-degradation, the long-term residue of waste has caused serious environmental pollution. Poly- $\beta$-hydroxybutyrate (PHB) and its soluble substances are similar to the petroleum plastic materials. It is considered to be a good substitute for the traditional plastic.. However, the low production efficiency and high production cost of PHB are the main problems that preclude its large-scale industrial production. The synthesis of PHB and high added value products will reduce the production cost of PHB. Ectoine (1,4,5,6-tetrahydro-2-methyl-4-pyrimidinecarboxylic acid, Ect) is one of the osmotic pressure compensation solute that produced by microorganism. It has a high commercial additional value. The preparation of PHB and Ectoine in a fermentation period by using PHB/Ect co-production technology shows great potential to promote industrial production and commercial applications of PHB and Ectoine. It has received more and more attention. In this article, the present situation and problems of $\mathrm{PHB} /$ Ect co-production were revealed. It aims to reduce production costs, improve production efficiency in PHB/Ect co-production.
\end{abstract}

\section{Introduction}

At present, the use of petrochemical industry plastics has exceeded 2 tons, and the annual growth rate was 5\% [1]. Because of its non-degradation, the long-term residue of waste has caused serious environmental pollution. Furthermore, petroleum is a non renewable resource, with its exploitation, the global storage is rapidly reduced. The new plastic material can replace the petrochemical industry plastics. It has become the research hotspot in the material science field and received the widespread attention [2].

Poly- $\beta$-hydroxybutyrate (PHB) and its soluble substances are considered to be a good substitute for the traditional plastic as its property of complete biodegradation and biocompatibility [3]. However, the low production efficiency and high production cost of PHB are the main problems that preclude its large-scale industrial production.

Ectoine (1,4,5,6-tetrahydro-2-methyl-4-pyrimidinecarboxylic acid, Ect) is one of the osmotic pressure compensation solute that accumulates in cells in the form of particles [4]. It can be widely used in many fields. It has a high commercial additional value. The co-production of Ectoine with high additional value production by using co-production technology of microorganism fermentation to synthesized PHB and ectoine (PHB/Ect) shows great potential to reduce production costs of PHB.

However, there are several limiting factors in PHB/Ect co-production system. High concentration $\mathrm{NaCl}$ inhibited the growth of cells and the synthesis of PHB [5]. Ectoine, which was synthetized at log phase, was degraded at stationary phase (PHB synthesis stage) [6]. co-production strains could not tolerate the osmotic down shock condition and thus could not be carried out "induction synthesis-down shock release" $\mathrm{PHB} /$ Ect co-production process with energy-conservation and environment-protection. 
In order to solve the problems in the current PHB/Ect co-production system, co-production strains should be selected, co-production fermentation strategy should be optimized. The establishment of low cost and high efficiency of PHB/Ect co-production and extraction system is an effective way to improve the economic benefit.

\section{PHB}

PHB is a biodegradable polymer polyester. It is widely used in industrial, pharmaceutical and agricultural areas. At present, PHB is mainly prepared by microbial fermentation with the price $\$ 4 / \mathrm{Kg}$, while the price of traditional petrochemical plastics is below $\$ 1 / \mathrm{Kg}$ [7]. High production costs restrict the industrialization production and application of PHB [8].

Application of PHB in the field of industrial packaging materials. Traditional plastic mainly comes from petroleum products. It degrades slowly and exists in the natural environment for a long time and thus causes serious pollution to the environment. PHB can be widely used as biodegradable plastic because of its bio-degradation. It reduced the "white pollution" caused by the traditional plastic. However, as its high production cost, PHB has not been applied in the field of industrial packaging materials.

Application of PHB in agricultural fields. In agricultural production, the application of agricultural plastic film can improve soil temperature, inhibit the growth of weeds. But the large scale waste film caused serious soil pollution. The degradable plastic film made from PHB can greatly reduce environmental pollution and soil. PHB can also be made into long acting preparation of bio-fertilizer, herbicides, pesticides and sustained release carrier. It is more conducive to the growth of crops [9].

\section{Ectoine}

Ectoine is an osmotic pressure compensatory solute. It is synthesized by Halomonas and other microorganisms in a hypertonic environment. As a stabilizing agent of cell, protein, nucleic acid and other biological products, ectoine can be widely used as cell protective agent, stabilizing agent of biological agents, and other fields $[4,10,11]$. It has a high commercial additional value. Therefore, an efficient production method of ectoine is also in great demand. Ectoine is mainly synthesized by microbial fermentation with low yield and high cost. The price of Ectoine with the purity of $95 \%$ made by German Bitop company was 440 yuan /g, while that with the purity of $98 \%$ made by American Santa Cruz company was 39428 yuan/g. Therefore, Ectoine has a high commercial additional value. Choi and Galinski used microbial fermentation to synthesize PHB and Ectoine (PHB/Ect) [12,13]. The co-production of high added value products Ectoine can effectively balance the economic cost of PHB synthesis.

\section{The co-production of $\mathrm{PHB}$ and Ectoine.}

PHB/Ect co-production strains. It has been reported that there are a lot of PHB/Ect co-production strains such as E. Halochloris [13], M. Marina and M. Terricola [14], M. Halotoleran and M. Solikamskensis [15], H. Campaniensis (5AGT) [16], H. Campaniensis [17], H. Elongata, H. Halodenitrificans, H. Haloneurihalina, H. Salina [18], H. Boliviensis [19] and etc. The moderately halophilic bacteria was the mostly species in the microorganism used in PHB/Ect co-production. Many species of Halomonas genus has been used in this research. However, the research results showed that the PHB/Ect was not effective in solving the problems of low efficiency, high production cost and high cost [19].

\section{The effects of PHB/Ect co-production.}

The effect of $\mathrm{NaCl}$ on PHB/Ect co-production. At present, the research on PHB/Ect co-production is concentrated in the ectoine-non-secreting strain. In a certain range of $\mathrm{NaCl}$, the synthesis amount of ectoine was positively correlated with the concentration of $\mathrm{NaCl}$ [20]. However, high concentration $\mathrm{NaCl}$ inhibited the cell growth, the synthesis of PHB and the degradation of ectoine 
at stationary phase(The stage of PHB synthesis) which was synthetized at log phase. It was the main limiting factor of cost and efficiency of PHB/Ect cosynthesis [19]. It was proposed that ectoine-secreting strain $H$. salina could be used for PHB/Ect co-production. Within a certain range of $\mathrm{NaCl}$ concentration, the total synthetic amount of ectoine was not affected by $\mathrm{NaCl}$ concentration and the secretion amount of ectoine was inversely proportional to $\mathrm{NaCl}$ concentration. It was realized that under the condition of low $\mathrm{NaCl}$ concentration $(30 \mathrm{~g} / \mathrm{L}), \mathrm{PHB}$ and ectoine were co-synthesized efficiently [21].

The effect of carbon source on PHB/Ect co-production. The most suitable carbon sources for PHB and ectoine synthesis are different. So the carbon source of the medium significantly effected on $\mathrm{PHB} /$ Ect co-production. Using glucose as carbon source, the synthetic amount of PHB was markedly improved, while monosodium glutamate was benefit for ectoine synthesis. Using glucose and sodium glutamate as the mixed carbon source, the synthetic amount of PHB/Ect co-production should be improved [19, 21].

The effect of nitrogen and phosphorus concentration on PHB/Ect co-production. Appropriate high $\mathrm{C} / \mathrm{N}$ ratio is benefit for PHB synthesis. Ectoine was synthesized at log phase and degraded at stationary phase (PHB synthesis stage) as carbon and nitrogen source for host [22]. The most suitable nitrogen and phosphorus concentrations in PHB and ectoine synthesis are not consistent. Limiting nitrogen and phosphorus concentrations could promote PHB synthesis while lead to the degradation of ectoine [18].

The effect of extraction method on PHB/Ect co-production product. PHB was conventionally extracted by chloroform after breaking cells by sodium hypochlorite ( $\mathrm{pH} 9.8$ ). However, ectoine is not stable at $\mathrm{pH}$ greater than 9. So this extraction method will cause the destruction of ectoine structure. Using the method of "down shock and water extraction", the above problems could be solved and the environmental pollution caused by the use of organic solvents could be avoided. But the number of viable cells decreased with the increase of the osmotic pressure difference. It indicated that down shock could result in cell rupture and die [23]. Sauer synthesized ectoine by "Bacterial Milking" using low osmotic pressure tolerance and non-ectoine-secreting strain $H$. Elongata. It realized the cyclic preparation and extraction of bacterial milking. With the cyclic preparation system, ectoine synthesis was not limited by the threshold of intracellular ectoine concentration and the preparation efficiency of ectoine was increased a lot. Ectoine-secreting strain $H$. venusta DSM 4743 was low osmotic pressure tolerance strain. It could also be used in "Bacterial Milking" to synthesize ectoine. It is supposed that low osmotic pressure tolerance and ectoine-secreting strain should realize the cyclic preparation and extraction of $\mathrm{PHB} /$ Ect co-production products [24].

\section{Conclusions}

In the present studies, it is difficult to co-synthesize PHB and ectoine with low cost and high yield. In order to solve the problems in the current $\mathrm{PHB} / \mathrm{Ect}$ co-production system, ectoine-secreting strain should be selected to co-synthesize PHB and ectoine. After optimizing co-production conditions and fermentation strategy, a PHB/Ect co-production and extraction system should be established and thus the cost of PHB/Ect co-production should be reduced and the economic benefit should be improved.

\section{References}

[1] G.W. Huisman, E. Wonink, et al: Journal of Biology and Chemistry Vol. 266 (1991), p. 2191

[2] A. Timm, A. Steinbuchel: European Journal of Biochemistry Vol. 209 (1992), p. 15.

[3] M. Lemoigne: Bull Soc. Chim. Biol. Vol. 8 (1926), p. 770.

[4] G. Lippert, E.A. Galinski: Appl. Microbiol. Biotechnol. Vol. 37 (1992), p. 61.

[5] A.J. Anderson, E.A. Dawes: Microbiol. Rev. Vol. 54 (1990), p. 450. 
[6] W. Babel, J.U. Ackermann, U. Breuer: Adv. Biochem. Eng. Biotechnol. 71 (Biopolyesters), Springer, Berlin, (2001), p. 125.

[7] E.Waltz: Nat. Biotechnol. Vol. 26 (2008), p.851.

[8] T. Keshavarz, I. Roy: Curr. Opin. Microbiol.Vol. 13 (2010), p.321.

[9] D. Kadouri, E. Jurkevitch, Y. Okon and et al: Critical Reviews in Microbiology Vol. 31 (2005), p.55.

[10] P. Louis, H.G. Trüper and E.A. Galinski: Appl. Microbiol. Biotechnol. 684.

[11] D.T. Welsh: FEMS Microbiol Rev. Vol. 24 (2000), p. 263.

[12] J.L. Cho and S.Y. Lee: Bioprocess Engineering Vol. 17 (1997), p. 335.

[13] E.A. Galinski and R.M. Herzog: Arch. Microbiol.Vol. 153 (1990), p. 607.

[14] N.V. Doronia, Y.A. Trotsenko and T.P. Tourova: International Journal of Systematic and Evolutionary Microbiology Vol. 50 (2000), p.1849.

[15] N.V. Doronia, M.N. Poroshina, E.N. Kaparullina and et al.:Systematic and Applied Microbiology Vol. 36 (2013), p.148.

[16] I. Romano, L. Lama, B. Nicolaus, Poli A and et al.: J. Gen. Appl. Microbiol. Vol. 52 (2006), p.339.

[17] G. Strazzullo, A. Gambacorta, F.M. Vella and et al.: World J. Microbiol. Biotechnol. Vol. 24 (2008), p. 1513.

[18] G. Mothes, T. Schubert, H. Harms and T. Mawkow: Eng. Life Sci. Vol. 8 (2008), p. 658.

[19] H. Guzmán, D. Van-Thuoc, J. Martín and et al.: Appl. Microbiol. Biotechnol. Vol. 84 (2009), p. 1069.

[20] M.I. Calderón, V. Carmen, R. Fernando and et al.: Microbiology Vol. 150 (2004), p. 3051.

[21] Q. Chen, L.H. Zhang, X.L. Li, S. Liu and D. Li: Process Biochemistry Vol. 49 (2014), p. 33.

[22] K. Schwibbert, A. Marin-Sanguino and et al.: Environ. Microbiol. Vol. 13 (2011), p. 1973.

[23] Q. Chen, T. Wang, J. Zhao and et al.: Advanced Materials Research Vol. 955-959 (2014), p. 3377.

[24] X. Zheng, H. Ma, X. Yan and L. Zhang: Microbiology China Vol. 37 (2010), p. 1090. 